\title{
Fabricating nanostructures on fused silica using femtosecond infrared pulses combined with sub-nanojoule ultraviolet pulses
}

\author{
Xiaoming Yu, ${ }^{1}$ Zenghu Chang, ${ }^{2}$ P. B. Corkum,${ }^{3,4}$ and Shuting Lei ${ }^{1, *}$ \\ ${ }^{1}$ Industrial and Manufacturing Systems Engineering, Kansas State University, Manhattan, Kansas 66506, USA \\ ${ }^{2}$ CREOL and Department of Physics, University of Central Florida, Orlando, Florida 32816, USA \\ ${ }^{3}$ National Research Council of Canada, Ottawa, Ontario K1A OR6, Canada \\ ${ }^{4}$ University of Ottawa, Ottawa, Ontario K1N 6N5, Canada \\ ${ }^{*}$ Corresponding author: lei@ksu.edu
}

Received July 29, 2014; revised August 27, 2014; accepted August 28, 2014;

posted August 29, 2014 (Doc. ID 219994); published September 24, 2014

\begin{abstract}
Circular craters with diameters of $500 \mathrm{~nm}$ are fabricated on the surface of fused silica by femtosecond ultravioletinfrared (UV-IR) pulse trains with $0.8 \mathrm{~nJ}$ UV pulse energy. UV damage thresholds at different IR energies and UV-IR delays are measured. Diameters and depths of the ablated craters can be modified by adding the IR pulse and varying the UV-IR delays. These results demonstrate the feasibility of nanomachining using short wavelength lasers with pulse energy far below normal damage thresholds. (c) 2014 Optical Society of America

OCIS codes: (320.2250) Femtosecond phenomena; (240.4350) Nonlinear optics at surfaces; (260.7190) Ultraviolet; (220.4241) Nanostructure fabrication.

http://dx.doi.org/10.1364/OL.39.005638
\end{abstract}

Femtosecond lasers have been used to fabricate various types of microstructures, such as waveguides, microfluidic networks, and three-dimensional (3D) data storage devices [1-3] . However, with conventional femtosecond systems operating at infrared wavelengths, fabricating these structures at nanometer scales requires high numerical aperture (NA) optics [4,5], precise control of pulse energy [6], or additional material processing steps [7]. Alternatively, by focusing short wavelength beams, such as ultraviolet (UV) and soft x-ray beams, features with sizes ranging from 80 to $600 \mathrm{~nm}$ have been fabricated [ $\underline{8}-10]$. While increasing energy output is still an ongoing research in generating short wavelength laser pulses, and indeed XUV pulses with $100 \mathrm{~nJ}$ have been reported [11], a machining technique requiring low pulse energies is still desired for practical applications, especially because of the low conversion efficiency in obtaining these beams. Our previous results show that by a combination of UV and IR beams, nanoscale features can be fabricated on fused silica with the UV pulse energy at only $10 \%$ of its normal value [12]. However, only line-shaped damage is achieved because of imperfect UV beam quality.

In this Letter, we apply a home-built spatial filter to improve the UV beam quality, and fabricate circular craters with diameters of $500 \mathrm{~nm}$ on the surface of fused silica, with the minimal UV pulse energy of $0.8 \mathrm{~nJ}$ using a UV-IR pulse train. These results demonstrate the feasibility of nanomachining using short wavelength lasers, even with their pulse energy far below normal damage thresholds.

Figure 1 shows the experimental setup. Synchronized femtosecond UV (266 nm) and IR (800 nm) pulses are generated from the same Ti:sapphire laser that operates at a repetition rate of $1 \mathrm{kHz}$ and delivers $60 \mathrm{fs}$ (full width at half-maximum, FWHM) IR pulses. The UV beam (estimated pulse duration 70 fs FWHM), generated from third-harmonic generation (THG) [1ㅡ] , first goes through a spatial filter which consists of two thin lenses
(L1, $f=500 \mathrm{~mm}$ and $\mathrm{L} 2, f=1 \mathrm{~m}$ ), and a pinhole drilled through a borosilicate microscope cover glass (thickness $150 \mu \mathrm{m})$ by another IR beam. The estimated UV pulse duration after L2 is 85 fs because of dispersion of the two lenses. The diameter of the pinhole matches the calculated focal spot size of L1. Lenses L1 and L2 also form a $2 \times$ beam expander so that the filtered UV beam with a smooth Gaussian profile overfills the input aperture of the reflecting objective (RO, Edmund, $0.5 \mathrm{NA}$, working

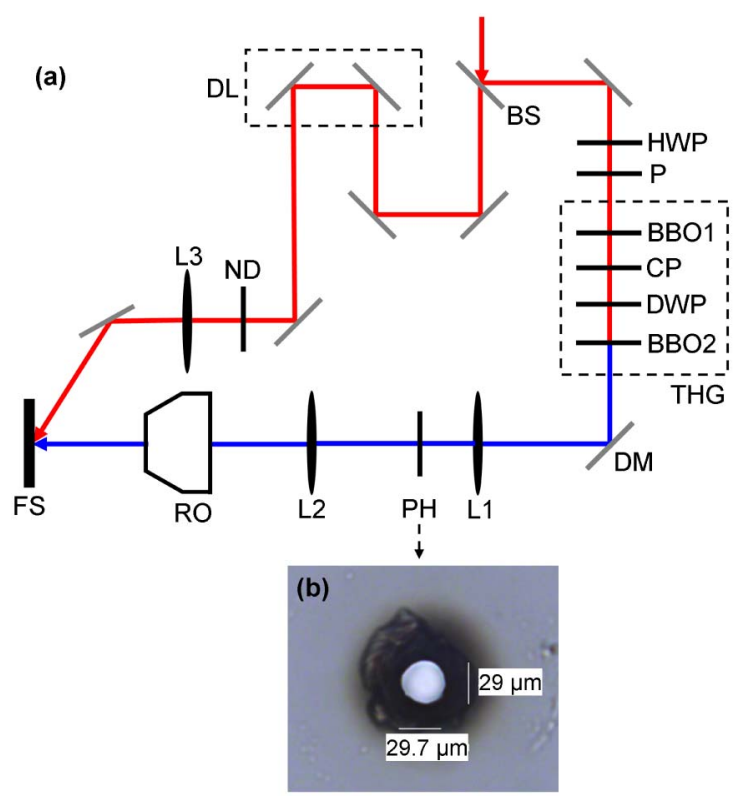

Fig. 1. (a) Experimental setup. BS, beamsplitter; HWP, halfwave plate; $\mathrm{P}$, polarizer; $\mathrm{BBO}, \beta$-barium borate; $\mathrm{CP}$, calcite plate; DWP, 400/800 nm dual wave plate; THG, third harmonic generate kit; DM, dichroic mirror; L1-L3, plano-convex lenses; $\mathrm{PH}$, pinhole; RO, reflecting objective; FS, fused silica sample; DL, delay line; and ND, neutral density filter. (b) Optical image of a pinhole used in the experiments. 
distance $23.2 \mathrm{~mm}$ ). The UV beam is then focused by the RO perpendicularly onto a fused silica sample (Corning 7980), with an estimated focal spot diameter of $0.64 \mu \mathrm{m}$. The sample is mounted on a motorized three-axis stage. The IR beam ( 60 fs FWHM) goes through a mechanical delay line and then is focused by L3 $(f=200 \mathrm{~mm})$ to the sample at a $\sim 45^{\circ}$ incidence angle. The IR spot size (observed from a CCD camera) is $20 \mu \mathrm{m} \times 15 \mu \mathrm{m}$ because of the oblique angle. The CCD camera monitoring the sample surface ensures that the UV spot remains at the center of the IR spot. The energy of the UV beam is controlled by a half-wave plate and a polarizer placed before the THG crystals, while the energy of the IR beam is controlled by a rotating neutral density (ND) filter. Both UV and IR energies are measured in front of the sample. The energy fluctuations of the UV and IR beams are within $5 \%$ and $1 \%$, respectively. For temporal overlapping, multiple-shot UV damage threshold is measured at different delays (temporal step $60 \mathrm{fs}$ ) at a fixed IR pulse energy below its damage threshold and a slow sample moving speed of $0.4 \mathrm{~mm} / \mathrm{s}$, and the optimal delay ( $60 \mathrm{fs}$ ) is determined when the lowest UV damage threshold is observed [13]. In the following single-shot measurements, the sample moves at a speed of $20 \mathrm{~mm} / \mathrm{s}$, ensuring that each UV-IR pulse pair irradiates at a fresh site. In all the cases no visual damage is seen with the IR beam alone.

At a certain delay and IR pulse energy, the single-shot UV damage threshold is determined as the lowest UV energy with which damage is observed using a scanning electron microscope (SEM). Twelve UV energies ranging from 0.4 to $19 \mathrm{~nJ}$ are used, and the results with five delays and three IR energies are shown in Fig. 2. The UV pulses arrive at the sample surface before and after the IR pulses at positive and negative delays, respectively. Similar curves have been reported, and can be explained in terms of free electron generation and defect states formation [12]. Theoretically, in ultrafast laser ablation of dielectrics, damage is formed when free electron density

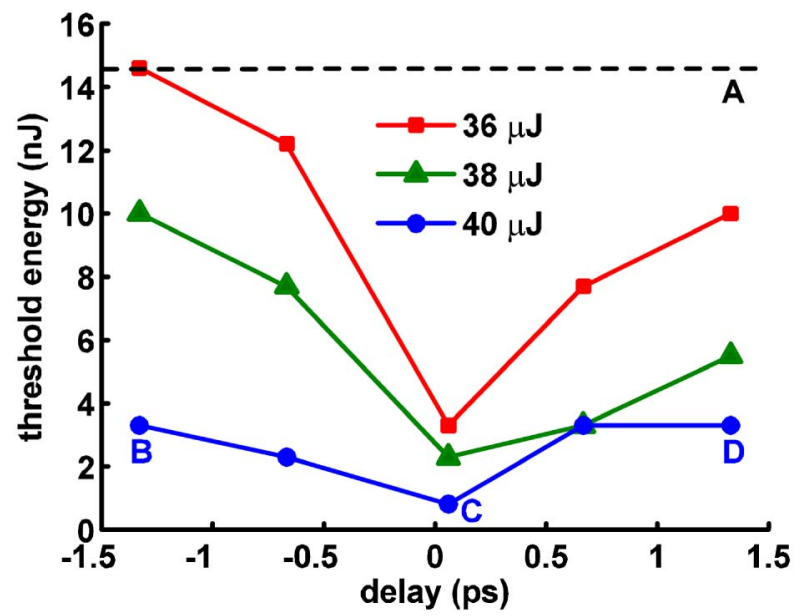

Fig. 2. UV damage threshold energy measured at different IR energies and delays. The black dashed line (A) indicates UV only threshold. SEM images corresponding to conditions A-D are shown in Figs. 3(a)-3(d), respectively. Square, triangular, and circular dots are experimental data. Solid lines are a guide to the eye. reaches a critical density. At positive delays, free electrons are first generated by the UV pulse through multiphoton ionization. These electrons either absorb energy from the following IR pulse at short delays $(<300 \mathrm{fs})$ or first decay into self-trapped excitons and then are re-ionized by the IR pulse at long delays ( $>1 \mathrm{ps})$ [14]. The result in both cases is a rapid increase in free electron density because of avalanche ionization during the IR pulse duration; eventually this density reaches the critical density, and damage is formed. With the help of the IR pulse, only a small number of free electrons (generated by the UV pulse) are needed to act as "seed electrons," and therefore the UV damage threshold is reduced. At negative delays, a similar process occurs except that the UV and IR pulses switch their roles.

The major difference of the results shown in Fig. 2 from our previous results is reduced UV threshold energies, for both the UV only and the pulse train case. Specifically, the UV only threshold is reduced from 64 to $14.6 \mathrm{~nJ}$, and the lowest UV threshold when combined with an IR pulse is reduced from 6 to 0.8 nJ. This reduction in threshold energy is attributed to the smaller focal spot and therefore smaller ablation area (shown below). Special attention is given to the case with $40 \mu \mathrm{J}$ IR energy, with which the UV damage threshold is reduced to $0.8 \mathrm{~nJ}$, only $5.5 \%$ of the normal threshold value. To the best of our knowledge, it is the first time that such low energy is used in femtosecond ablation of dielectrics. It should be noted that, even with the improved UV beam, the damage threshold energy $(14.6 \mathrm{~nJ})$ is still $\sim 5$ times higher than estimated $(2.8 \mathrm{~nJ})$ for a focal diameter of $0.64 \mu \mathrm{m}$ [15], perhaps because of a Bessel-like instead of a Gaussian profile of the UV focus (shown below in Fig. 3) [16]. Therefore, nanoablation with even lower pulse energy is possible by replacing the reflecting objective with a transmitting objective, which generates an Airy pattern with more energy concentrated in the central spot compared to the Bessel-like pattern. It is worth noting that, although Fig. 2 emphasizes the reduction of UV damage threshold, these data can also be interpreted as a reduction of IR threshold with the help of UV pulses. For

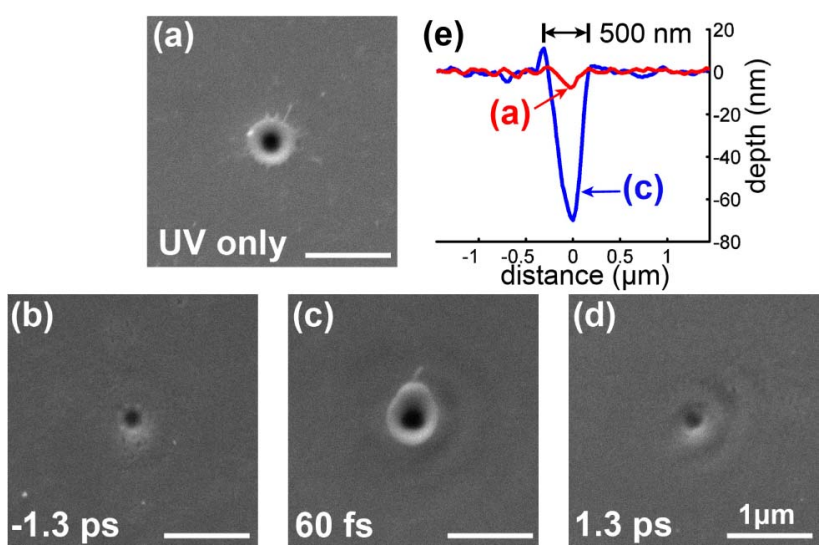

Fig. 3. (a)-(d) SEM images of the damage spots with UV beam only, UV-IR pulse train at $-1.3 \mathrm{ps}, 60 \mathrm{fs}$, and $1.3 \mathrm{ps}$ delay, respectively. (a)-(d) correspond to the conditions labeled A-D in Fig. 2, respectively. (e) Typical cross sections of the damage in (a) and (c) measured with an atomic force microscope (AFM). 
example, Point B in Fig. 2 indicates that, with $3.5 \mathrm{~nJ}$ UV pulse energy and $-1.3 \mathrm{ps}$ delay, the IR threshold is reduced from $42 \mu \mathrm{J}$ (IR only threshold) to $40 \mu \mathrm{J}$.

Figures 3(a)-3(d) show SEM images of the damage spots corresponding to A-D in Fig. 2, respectively, and typical cross-sections for Figs. 3(a) and 3(c) are shown in Fig. 3(e). Craters with circular shapes are achieved with the aforementioned beam-shaping technique for both UV only [Fig. 3(a)] and the pulse train case [Figs. 3(b)-3(d)]. An increase in crater depth is observed: from $7 \mathrm{~nm}$ with the UV beam alone [Fig. 3(a)] to $70 \mathrm{~nm}$ with the UV-IR pulse train at a 60 fs delay [Fig. 3(c)], while the damage size on the surface remains similar. In addition, Figs. 3(b) and 3(d) show that craters fabricated with a pulse train tend to be smaller at longer delays. Although these two effects, namely the increase in ablation depth with pulse trains and the reduction in feature size at long delays, have been reported $[\underline{17}, \underline{18}]$; to the best of our knowledge, it is the first time to show these effects in nanomachining; thus it indicates the possibility of controlling the size of fabricated nanostructures through pulse delays. The ripple structures adjacent to the central craters in Figs. 3(c) and 3(d) are attributed to the Bessel-like profile of the UV focus [16]. Combining with the IR beam enhances ablation effects and therefore reveals the otherwise unseen structures with the UV beam only [Fig. 3(a)]. These structures can be removed when a transmitting objective lens is used (see the paragraph above).

In summary, through the fabricated $500 \mathrm{~nm}$ features on fused silica by UV-IR pulse trains with $0.8 \mathrm{~nJ}$ UV pulse energy, we demonstrate the feasibility of nanomachining using even shorter wavelength (XUV, $\mathrm{x}$ ray) lasers with pulse energies far below the normal damage threshold. The addition of long wavelength pulses can improve ablation rates, and resultant damage shapes can be further controlled by changing the delays of the pulse trains.

The authors thank Prof. Daniel A. Higgins, Prof. Jun Li, $\mathrm{Hao} \mathrm{Xu}$ and Yiqun Yang for their assistance in AFM imaging. This material is based on work supported by the Army Research Office and the National Science Foundation under Grant Number CMMI 1131627.

\section{References}

1. R. R. Gattass and E. Mazur, Nat. Photonics 2, 219 (2008).

2. K. Sugioka and Y. Cheng, Light Sci. Appl. 3, e149 (2014).

3. M. H. Hong, B. Luk'yanchuk, S. M. Huang, T. S. Ong, L. H. Van, and T. C. Chong, Appl. Phys. A 79, 791 (2004).

4. S. I. Kudryashov, G. Mourou, A. Joglekar, J. F. Herbstman, and A. J. Hunt, Appl. Phys. Lett. 91, 141111 (2007).

5. J. M. Fernández-Pradas, C. Florian, F. Caballero-Lucas, J. L. Morenza, and P. Serra, Appl. Surf. Sci. 278, 185 (2013).

6. A. P. Joglekar, H.-H. Liu, E. Meyhöfer, G. Mourou, and A. J. Hunt, Proc. Natl. Acad. Sci. USA 101, 5856 (2004).

7. Y. Liao, Y. Shen, L. Qiao, D. Chen, Y. Cheng, K. Sugioka, and K. Midorikawa, Opt. Lett. 38, 187 (2013).

8. J. Békési, J.-H. Klein-Wiele, and P. Simon, Appl. Phys. A 76, 355 (2003).

9. G. Vaschenko, A. G. Etxarri, C. S. Menoni, J. J. Rocca, O. Hemberg, S. Bloom, W. Chao, E. H. Anderson, D. T. Attwood, Y. Lu, and B. Parkinson, Opt. Lett. 31, 3615 (2006).

10. M. Dubov, I. Bennion, D. N. Nikogosyan, P. Bolger, and A. V. Zayats, J. Opt. A 10, 025305 (2008).

11. Y. Wu, E. Cunningham, H. Zang, J. Li, M. Chini, X. Wang, Y. Wang, K. Zhao, and Z. Chang, Appl. Phys. Lett. 102, 201104 (2013).

12. X. Yu, Q. Bian, Z. Chang, P. B. Corkum, and S. Lei, Opt. Express 21, 24185 (2013).

13. X. Yu, Q. Bian, B. Zhao, Z. Chang, P. B. Corkum, and S. Lei, Appl. Phys. Lett. 102, 101111 (2013).

14. D. Grojo, M. Gertsvolf, S. Lei, T. Barillot, D. M. Rayner, and P. B. Corkum, Phys. Rev. B 81, 212301 (2010).

15. T. Q. Jia, H. X. Chen, M. Huang, F. L. Zhao, X. X. Li, S. Z. Xu, H. Y. Sun, D. H. Feng, C. B. Li, X. F. Wang, R. X. Li, Z. Z. Xu, X. K. He, and H. Kuroda, Phys. Rev. B 73, 054105 (2006).

16. R. M. Herman and T. A. Wiggins, J. Opt. Soc. Am. A 8, 932 (1991).

17. I. H. Chowdhury, X. Xu, and A. M. Weiner, Appl. Phys. A 83, 49 (2006).

18. S. Zoppel, R. Merz, J. Zehetner, and G. A. Reider, Appl. Phys. A 81, 847 (2005). 